\title{
Prevalence and profile of diabetes kidney disease according to different diagnostic criteria in type 2 diabetes mellitus patients
}

Sabrina Coelli, Ariana Aguiar Soares, Camila Kümmel Duarte, Ana Marina Moreira, Luiza Barboza de Souza, Andrea Carla Bauer, Themis Zelmanovitz, Sandra Pinho Silveiro

From 20th Brazilian Diabetes Society Congress

Porto Alegre, Brazil. 11-18 November 2015

\section{Background}

Diabetes kidney disease (DKD) is the worldwide leading cause of end-stage renal disease. Diagnostic criteria have been recently revised.

\section{Objective}

The aim of this study was to evaluate the prevalence and clinical profile of type $2 \mathrm{DM}$ patients according to the employed definition of DKD: previous diagnostic criteria as compared to the present one.

\section{Materials and methods}

566 type 2 DM outpatients from the Endocrine Unit ambulatory were included. DKD was defined by the presence of elevated urinary albumin excretion alone (UAE; $>14 \mathrm{mg} / \mathrm{l}$ ) - previous definition- or by the presence of elevated UAE and/or reduced $(<60 \mathrm{ml} / \mathrm{min} /$ $1.73 \mathrm{~m} 2$ ) glomerular filtration rate (GFR) -present definition.

\section{Results}

Mean age was $63 \pm 11$ yrs., $37 \%$ men, $86 \%$ white, $10 \%$ smokers, DM duration 16 yrs. When evaluated by elevated UAE only, $50 \%$ of the patients presented DKD. Table 1 shows the profile of these patients (Fig 1). When defined by both UAE and GFR, $57 \%$ presented DKD, and Table 2 shows the profile of these patients (Fig 2).

\begin{tabular}{llll}
\hline & $\begin{array}{l}\text { No DKD } \\
(\mathrm{N}=283)\end{array}$ & $\begin{array}{c}\text { DKD } \\
(\mathrm{N}=283)\end{array}$ & $\mathrm{P}$ \\
\hline Age $(\mathrm{y})$ & $63 \pm 10$ & $63 \pm 11$ & 0.758 \\
Duration of DM (y) & $16(2-42)$ & $16(2-52)$ & 0.490 \\
Gender $(\mathrm{men})$ & $82(29 \%)$ & $125(44 \%)$ & $<0.001$ \\
Ethnicity $(\mathrm{W} / \mathrm{B})$ & $248 / 35$ & $240 / 43$ & 0.172 \\
BMl $\left(\mathrm{kg} / \mathrm{m}^{2}\right)$ & $30 \pm 5$ & $31 \pm 5$ & 0.001 \\
SBP $(\mathrm{mmHg})$ & $130(80-205)$ & $140(80-230)$ & $<0.001$ \\
DBP $(\mathrm{mmHg})$ & $80(50-110)$ & $80(50-130)$ & 0.002 \\
HbA1c $(\%)$ & $8.0(5.1-14.8)$ & $8.2(4.3-15.2)$ & 0.048 \\
eGFR (ml/min/1.73 $\left.\mathrm{m}^{2}\right)$ & $97(19-157)$ & $81(7-160)$ & $<0.001$ \\
TG (mg/dl) & $137(28-696)$ & $153(36-2742)$ & 0.001 \\
HDL (mg/dl) & $40(23-94)$ & $38(19-87)$ & 0.014 \\
TC (mg/dl) & $161(45-281)$ & $165(64-350)$ & 0.664 \\
& & &
\end{tabular}

DKD: diabetic kidney disease: BMI: body mass index: SBP: systolic blood pressure: DBP diastolic blood prossure; HbA1c: glycated hemoglobin; eGFR: ostimated glomerular filtration; TG: triglycerides; TC: total cholesterol.

Figure 1 Clinical and laboratory characteristics of DKD patients according to the presence of elevated UAE only.

\footnotetext{
* Correspondence: sabrina.coelli@gmail.com

Universidade Federal do Rio Grande do Sul (UFRGS), Porto Alegre, Brazil
} 


\begin{tabular}{llll}
\hline & $\begin{array}{l}\text { No DKD } \\
(\mathrm{N}=246)\end{array}$ & $\begin{array}{c}\text { DKD } \\
(\mathrm{N}=320)\end{array}$ & $\mathrm{P}$ \\
\hline Age (years) & $62 \pm 10$ & $64 \pm 11$ & 0.033 \\
Duration of DM (y) & $15(2-42)$ & $16(2-52)$ & 0.176 \\
Gender $(\mathrm{man})$ & $53(21 \%)$ & $154(48 \%)$ & $<0.001$ \\
Ethnicity(W/B) & $215 / 31$ & $273 / 47$ & 0.271 \\
BMI $\left(\mathrm{kg} / \mathrm{m}^{2}\right)$ & $30 \pm 5$ & $31 \pm 5$ & 0.079 \\
SBP $(\mathrm{mmHg})$ & $130(80-205)$ & $140(80-240)$ & 0.001 \\
DBP $(\mathrm{mmHg})$ & $80(50-110)$ & $80(50-130)$ & 0.020 \\
HbA1c $(\%)$ & $8.0(5.1-14.8)$ & $8.0(4.3-15.2)$ & 0.175 \\
eGFR $\left(\mathrm{ml} / \mathrm{min} / 1.73 \mathrm{~m}^{2}\right)$ & $100(61-157)$ & $75(7-160)$ & $<0.001$ \\
TG $(\mathrm{mg} / \mathrm{dl})$ & $138(28-696)$ & $151(36-2742)$ & 0.006 \\
HDL $(\mathrm{mg} / \mathrm{dl})$ & $41(23-94)$ & $38(19-87)$ & 0.001 \\
TC $(\mathrm{mg} / \mathrm{dl})$ & $164(94-281)$ & $162(45-350)$ & 0.434 \\
& & & \\
\hline
\end{tabular}

DKD: diabetic kidney disease: BMI: body mass index; SBP: systolic blood pressure: DBP diastolic blood pressure: HbAic: glycated hemoglobin; eGFR: estimated glomerular filtration; TG: triglyceridos: TC: total cholosterol.

Figure 2 Clinical and laboratory characteristics of DKD patients according to the presence of elevated UAE and/or reduced GFR.

\section{Conclusion}

DKD cases would be missed if only UAE is taken into account.

Published: 11 November 2015

doi:10.1186/1758-5996-7-S1-A28

Cite this article as: Coelli et al:: Prevalence and profile of diabetes kidney disease according to different diagnostic criteria in type 2 diabetes mellitus patients. Diabetology \& Metabolic Syndrome 20157

(Suppl 1):A28.

Submit your next manuscript to BioMed Central and take full advantage of:

- Convenient online submission

- Thorough peer review

- No space constraints or color figure charges

- Immediate publication on acceptance

- Inclusion in PubMed, CAS, Scopus and Google Scholar

- Research which is freely available for redistribution

Submit your manuscript at www.biomedcentral.com/submit
C Biomed Central 